\title{
Learning Automata Based Algorithms for Mapping of a Class of Independent Tasks over Highly Heterogeneous Grids
}

\author{
S. Ghanbari and M. R. Meybodi \\ Soft Computing Laboratory \\ Computer Engineering Department and Information Technology \\ Amirkabir University \\ Tehran Iran \\ Email: saeed_ghanbari@yahoo.com,meybodi@ce.aut.ac.ir
}

\begin{abstract}
The computational grid provides a platform for exploiting various computational resources over wide area networks. One of the concerns in implementing computational grid environment is how to effectively map tasks onto resources in order to gain high utilization in the highly heterogeneous environment of the grid. In this paper, three algorithms for task mapping based on learning automata are introduced. To show the effectiveness of the proposed algorithms, computer simulations have been conducted. The results of experiments show that the proposed algorithms outperform two best existing mapping algorithms when the heterogeneity of the environment is very high.
\end{abstract}

\section{Introduction}

Owing to advances in computational infrastructure and networking technology, construction of large-scale high performance distributed computing environment, known as computational grid, is now possible. Computational Grid enables the sharing, selection, and aggregation of geographically distributed heterogeneous resources for solving large scale problems in science, engineering and commerce. Numerous efforts have been exerted focusing on various aspects of grid computing including resource specifications, information services, allocation, and security issues. A critical issue to meeting the computational requirements on the grid is the scheduling.

Ensuring a favorable efficiency over computational grid is not a straightforward task, where a number of issues make scheduling challenging even for highly parallel applications. Resources on the grid are typically shared and undedicated so that the contention made by various applications results in dynamically fluctuating delays, capricious quality of services, and unpredictable behavior, which further complicate the scheduling. Regarding to these hurdles, the scheduling of applications on computational grids have become a major concern of multitude efforts in recent years [9]. 
In mixed-machine heterogeneous computing (HC) environments like computational grids, based on application model characterization, platform model characterization and mapping strategy characterization, there are various definitions for scheduling[6]. Ideal sorts of applications for computational grid are those composed of independent subtasks (called metatask), which subtasks can be executed in any order and there is no inter-task communication (i.e. totally parallel) [1][13]. There are many applications of such feature including data mining, massive searches (such as key breaking), parameter sweeps, Monte Carlo simulations[2], fractals calculations (such as Mandelbrot), and image manipulation applications (such as tomographic reconstruction[3]). Computational grid platform model consists of different high-performance machines, interconnected with high-speed links. Each machine executes a single task at a time (i.e. no multitasking) in the order to which the tasks are assigned. The size of the metatask and the number of machines in the HC suite are static and known beforehand. The matching of tasks to machines and scheduling the execution order of these tasks is referred to as mapping. The general problem of optimally mapping tasks to machines in an $\mathrm{HC}$ suite has been shown to be NP-complete[11].

In this paper, we present three algorithms based on learning automata for mapping metatask over HC. Through computer simulations we show that the proposed algorithms outperform the best existing mapping algorithms when the heterogeneity of the environment is very high.

This paper is organized as follows: Section 2 discusses the related works. Section 3 introduces the learning automata. Section 4 explains the model of the Grid and the definitions used in later sections. Section 5 introduces the proposed learning automata based algorithms. In Section 6, experimental results are discussed and section 7 is the conclusion.

\section{Related works}

Existing mapping algorithms can be categorized into two classes[4]: on-line mode (immediate) and batch mode. In on-line mode, a task is mapped onto a host as soon as it arrives at the scheduler. In the batch mode, tasks are collected into a set of tasks that is examined for mapping at certain intervals called mapping events. The independent set of tasks that is considered for mapping at the mapping events is called a metatask. The on-line mode is suitable for low arrival rate, while batch-mode algorithms can yield higher performance when the arrival rate of tasks is high because there will be a sufficient number of tasks to keep hosts busy between the mapping events, and scheduling is done according to the resource requirement information of all tasks in the set[4]. The objective of most mapping algorithms is to minimize makespan, where makespan is the time needed for completing the execution of a metatask Minimizing makespan yields to higher throughput.

Minimum Completion Time (MCT), Minimum Execution Time (MET), Dual, and $k$-Percent Best (KPB) are among well known existing on-line mode heuristics[5]. Reported batch mode heuristics are Min-Min, Max-Min, Genetic Algorithm (GA), Simulated Annealing (SA), Genetic Simulated Annealing (GSA), A ${ }^{*}$ search, 
Learning Automata Based Algorithms for Mapping of a Class of Independent Tasks over

Highly Heterogeneous Grids 3

Suffrage[5][4], and Relative Cost (RC) [7] . Experiments results from [5] show that among batch-mode heuristics, Min-Min and GA give lower makespan.[7] proposes Relative Cost (RC) heuristic which further outperforms both GA and Min-Min.

$\mathrm{RC}$ introduces two essential criteria for a high-quality mapping algorithm for heterogeneous computing systems: matching which is to better match the tasks and machines, and load balancing which is to better utilize the machines. It is shown that in order to minimize the makespan, matching and system utilization should be maximized, and an ideal algorithm should satisfy both criteria simultaneously. However, these design goals are in conflict with each other because mapping tasks to their first choice of machines may cause load imbalance. Therefore, the mapping problem is essentially a tradeoff between the two criteria. Two out of three proposed heuristics in this paper resolve a mapping by optimizing matching proximity and system utilization.

\section{Learning Automata}

Learning Automata are adaptive decision-making devices operating on unknown random environments. A Learning Automaton has a finite set of actions and each action has a certain probability (unknown to the automaton) of getting rewarded by the environment of the automaton. The aim is to learn to choose the optimal action (i.e. the action with the highest probability of being rewarded) through repeated interaction on the system. If the learning algorithm is chosen properly, then the iterative process of interacting on the environment can be made to result in selection of the optimal action. Figure 1 illustrates how a stochastic automaton works in feedback connection with a random environment. Learning Automata can be classified into two main families: fixed structure learning automata and variable structure learning automata (VSLA) [8]. In the following, the variable structure learning automata which will be used in this paper is described.

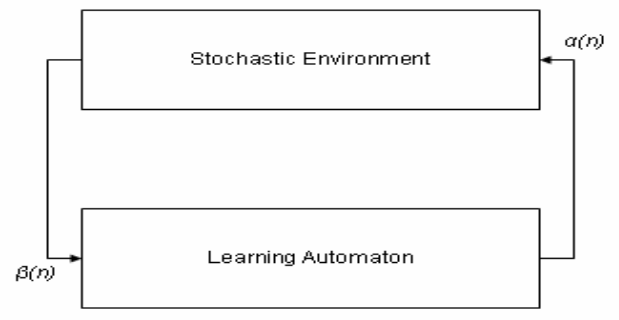

Fig. 1. The interaction between learning automata and environment

A VSLA is a quintuple $<\alpha, \beta, p, T(\alpha, \beta, p)>$, where $\alpha, \beta, p$ are an action set with $s$ actions, an environment response set and the probability set $p$ containing $s$ probabilities, each being the probability of performing every action in the current internal automaton state, respectively. If the response of the environment takes binary values learning automata model is P-model and if it takes finite output set with more than two elements that take values in the interval $[0,1]$, such a model is referred to as 
Q-model, and when the output of the environment is a continuous variable in the interval $[0,1]$, it is refer to as $\mathrm{S}$-model. The function of $T$ is the reinforcement algorithm, which modifies the action probability vector $p$ with respect to the performed action and received response. Assume $\beta \in[0,1]$. A general linear schema for updating action probabilities can be represented as follows. Let action $i$ be performed then:

$$
\begin{gathered}
p_{j}(n+1)=p_{j}(n)+\beta(n)\left[b /(r-1)-b p_{j}(n)\right]-[1-\beta(n)] a p_{j}(n) \forall j j \neq i \\
p_{i}(n+1)=p_{i}(n)-\beta(n) b p_{i}(n)+[1-\beta(n)] a\left[1-p_{i}(n)\right]
\end{gathered}
$$

where $a$ and $b$ are reward and penalty parameters. When $a=b$, the automaton is called $\mathrm{L}_{\mathrm{RP}}$. If $b=0$ the automaton is called $\mathrm{L}_{\mathrm{RI}}$ and if $0<b<<a<1$, the automaton is called $\mathrm{L}_{\mathrm{R \varepsilon}}$. For more Information about learning automata the reader may refer to [8].

\section{Simulation Model}

This section presents a general model of the computational Grid. Figure 2 shows the schematic representation of the environment. The environment consists of the heterogeneous suite of machines which will be used to execute the application. The scheduling system consists of the automata, and the model of the application and the $\mathrm{HC}$ suite of machines. The application and HC suite of machines are modeled as the estimate of the expected execution time for each task on each machine, which is known prior to the execution and contained within a $\tau \times \mu$ ETC (Expected Time to Compute) matrix, where $\tau$ is the number of tasks and $\mu$ is the number of machines. One row of the ETC matrix contains the estimated execution times for a given task on each machine. Similarly, one column of the ETC matrix consists of the estimated execution times of a given machine for each task in the metatask. Thus, for an arbitrary task $t_{i}$ and an arbitrary machine $m_{j}, E T C\left(t_{i}, m_{j}\right)$ is the estimated execution time of $t$ on $m_{j}$. The $E T C\left(t_{i}, m_{j}\right)$ entry could be assumed to include the time to move the executables and data associated with task $t_{i}$ from their known source to machine $m_{j}$. For cases when it is impossible to execute task $t_{i}$ on machine $m_{j}$ (e.g., if specialized hardware is needed), the value of $E T C\left(t_{i}, m_{j}\right)$ is set to infinity.

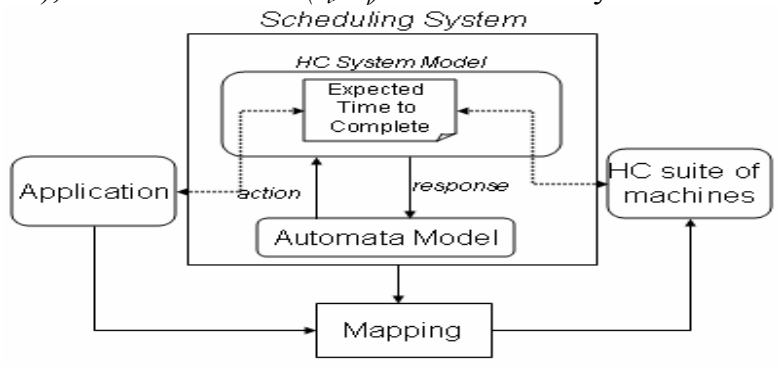


Learning Automata Based Algorithms for Mapping of a Class of Independent Tasks over Highly Heterogeneous Grids 5

Fig. 2. Model of the Grid

We define $\psi^{(n)}(i)=j$ as a general mapping from the task domain $i=1, \ldots, \tau$ to the machine domain $j=1, \ldots, \mu$ at iteration $n$. The load of each machine, which is denoted by $\theta^{(n)}(j)$, is defined as the time taken to execute all the assigned tasks:

$$
\theta^{(n)}(j)=\sum \operatorname{ETC}(k, j), j=\psi^{(n)}(k) 1 \leq k \leq \tau
$$

The maximum $\theta^{(n)}(j)$ value, over $1 \leq j \leq \mu$, is the metatask execution time, which is referred to as makespan denoted by $T_{\mu}^{(n)}$.

\section{Proposed Learning Automata Model}

The learning automata model is constructed by associating every task $s_{i}$ in the metatask with a variable structure learning automaton, which is represented by a 3 tuple $(\alpha(i), \beta(i), A(i))$. Each action of an automaton is associated with a machine, and since the tasks can be assigned to any of the $\mu$ machines ( $\mu$ : number of machines), the action set of all learning automata are identical. Therefore, for any task $\mathrm{s}_{\mathrm{i}}, 1 \leq i \leq \tau$ ( $\tau$ number of tasks), $\alpha(i)=m_{1}, m_{2}, \ldots, m_{\mu}$ and $\beta(i) \in[0,1]$, where $\beta(i)$ closer to 0 indicates that the action taken by the automaton of task $s_{i}$ is favorable to the system, and closer to 1 indicates an unfavorable response. Reinforcement scheme used to update action probabilities of learning automata is $\mathrm{L}_{\mathrm{RI}}$.

To determine the goodness of an action taken by an automaton, we propose three different algorithms. The first algorithm calculates $\beta(i)$ for each automaton $A(i)$ according to the reduction made in makespan and the load of the selected machine. The second and third algorithms calculate the goodness of an action based on improvement made in matching and load balancing.

\subsection{Algorithm No.1}

The algorithm No.1 (A1) determines the $\beta^{(n)}(i)$ at iteration $\mathrm{n}$ for each automaton $A(i)$ by considering makespan and load of the chosen machine. Algorithm A1 interprets the environment as P-model and therefore $\beta^{(n)}(i) \in\{0,1\}$. Makespan at iteration $n$ may be greater, less than, or equal to makespan at iteration $n$ - 1 . Similarly, load of the machine chosen by automaton $A(i)$ at iteration $n$ may be greater, less than, or equal to load of the machine chosen by the automaton at iteration $n$ - 1 . Therefore, regarding to makespan and the load of the chosen machine in two consecutive iterations, nine states are possible. To determine the $\beta^{(n)}(i)$, we associate a probability value to each nine possible state, which determines the probability of rewarding the 
chosen action. Probability zero means that the chosen action will be rewarded. Table 1 shows the values, where $D, U$ and $I$ stand for decrease, remaining unchanged, and increase, respectively.

\begin{tabular}{|c|c|c|}
\hline Makespan & Load of chosen machine & Rewarding probability \\
\hline$D$ & $D$ & 1 \\
\hline$D$ & $U$ & 0.875 \\
\hline$D$ & $I$ & 0.75 \\
\hline$U$ & $D$ & 0.625 \\
\hline$U$ & $U$ & 0.5 \\
\hline$U$ & $I$ & 0.375 \\
\hline$I$ & $D$ & 0.25 \\
\hline$I$ & $U$ & 0.125 \\
\hline$I$ & $I$ & 0 \\
\hline
\end{tabular}

Table 1. Reward probability associated with each state

At iteration $\mathrm{n}$, an automaton receives reward when the makespan and the load of chosen machine reduce, and, it receives penalty for other cases with probabilities as given in the table. Algorithm $\mathrm{Al}$ is suitable for situations that the information used to evaluate the environment response is the load of machines.

\subsection{Algorithm No.2}

Algorithm No.2 (A2) evaluates the response to the learning automata by considering two criteria: matching and system utilization. It is shown that to minimize the makespan, matching and system utilization must be maximized0. Matching of tasks and machines can be measured by a parameter, matching proximity, which is defined as follows:

$$
\eta=\frac{\sum_{1 \leq i \leq \tau} \operatorname{ETC}\left(i, \psi_{\min }(i)\right)}{\sum_{1 \leq i \leq \tau} \psi(i)}
$$

where $\eta \leq 1$ and $\psi_{\min }(i)$, is the ideal matching. Ideal matching is defined as executing every task on the machine with the shortest execution time. It is defined as follows:

$$
\psi_{\min }(i)=j \text { such that } \operatorname{ETC}(i, j)=\min _{1 \leq q \leq \mu} \operatorname{ETC}(i, q)
$$

when $\eta=1$, we have the ideal matching.

System utilization is defined as follows:

$$
\delta=\frac{\sum_{1 \leq i<\mu} E T C(i, \Psi \quad(i))}{\mu \times T_{\mu}}
$$

which $T_{\mu}$ is the makespan. When the system is completely balanced, $\delta=1$; otherwise $\delta<1$. 
Learning Automata Based Algorithms for Mapping of a Class of Independent Tasks over Highly Heterogeneous Grids 7

Algorithm A2 reduces the mapping problem to an optimization problem with matching proximity and system utilization as objective functions. Algorithm A2 interprets the environment as S-model; that is $\beta^{(n)}(i) \in[0,1]$.

To evaluate the contribution of each automaton to improving matching and system utilization, we define two parameters, partial contribution to matching(PCM) and partial contribution to load balancing(PCL). Input to each automaton is a linear combination of PCM (denoted by $\eta^{(n)}(i)$ ), and PCL (denoted by $\delta^{(n)}(i)$ ):

$$
\beta^{(n)}(i)=\eta^{(n)}(i) \lambda_{\eta}+\delta^{(n)}(i) \lambda_{\delta} \text { where } \lambda_{\eta}+\lambda_{\delta}=1
$$

$\lambda_{\eta}$ and $\lambda_{\delta}$ are weights associated with PCM and PCL, respectively.

PCM for each automaton $A(i)$ at iteration $n$ is evaluated as:

$$
\eta^{(n)}(i)=\frac{\operatorname{ETC}\left(i, \psi^{(n)}(i)\right)-\operatorname{ETC}\left(i, \psi_{\min }(i)\right)}{\operatorname{ETC}\left(i, \psi_{\text {max }}(i)\right)-\operatorname{ETC}\left(i, \psi_{\text {min }}(i)\right)}
$$

where $\psi_{\max }(i)$ is the worst matching which is defined as mapping each task to a machine with the longest execution time; it is defined below

$$
\psi_{\max }(i)=j \text { such that } \operatorname{ETC}(i, j)=\max _{1 \leq q \leq \mu} \operatorname{ETC}(i, q)
$$

The closer $\eta^{(n)}(i)$ to 0 , the more favorable the response from the environment as far as the matching is concerned. In the case that the automaton selects the machine with the worst matching, $\eta^{(n)}(i)$ is evaluated to 1 .

PCL for each automaton $A(i)$ at iteration $n$ is evaluated as:

$$
\partial^{(n)}(i)=\frac{\theta^{(n)}\left(\psi^{(n)}(i)\right)}{T_{\mu}^{(n)}}\left(1-e^{-1 / 2\left(\frac{\delta^{(n)}-1}{0.1}\right)}\right)
$$

The former part of the above expression is close to 0 when the chosen machine has a load less than the maximum load. Thus, the learning automata are encouraged to choose machines with low loads, thus, they are guided in a way to decrease the distance between the maximum load and the minimum load. The latter part of the expression is a Gaussian function. It gets closer to 0 as the system utilization increases; therefore, when the load is relatively balanced, PCL of each automaton is close to 0 . Unlike algorithm A1, algorithm A2 requires information about the estimation of execution time of each task on each machine. Therefore, algorithm A2 can be used if such information can be obtained.

\subsection{Algorithm No.3}

Algorithm No.3 (A3) interprets the environment as a Q-Model environment. Like algorithm A2, algorithm A3 uses matching proximity and system utilization as objective functions. PCL and PCM are evaluated in the same way as algorithm A2, 
and used to produce the environment response. But, in algorithm A3, PCL and PCM are interpreted as probabilities, where PCL determines the probability that the learning automaton receives unfavorable response as far as system utilization is concerned, and PCM determines the probability that the learning automaton receives unfavorable response as far as matching is concerned. The environment response is evaluated as below:

$$
\beta^{(n)}(i)=I_{\eta^{(n)}(i)} \lambda_{\eta}+I_{\delta^{(n)}(i)} \lambda_{\delta} \text { where } \lambda_{\eta}+\lambda_{\delta}=1
$$

$\lambda_{\eta}$ and $\lambda_{\delta}$ are the weights associated with PCM and PCL, respectively. $I_{\eta^{(n)}(i)}$ is an indicator function which returns 1 with the probability of $\eta^{(n)}(i)$ and 0 with the probability of $1-\eta^{(n)}(i) \cdot I_{\delta^{(n)}(i)}$ is also an indicator function which returns 1 with the probability of $\delta^{(n)}(i)$ and 0 with the probability of $1-\delta^{(n)}(i)$. Therefore, the input to each automaton $\beta(i)$ is in $\left\{0, \lambda_{\eta}, \lambda_{\sigma}, 1\right\}$. In contrast to algorithm A2, algorithm A3 evaluates environment response stochastically, which allows the learning automata to jump local minimums in their search space.

\section{Experiments}

In this section the proposed algorithms are tested and compared with Min-Min and $\mathrm{RC}$ because these two algorithms are the best existing algorithms. For the simulation studies, characteristics of the ETC matrices were varied in an attempt to represent a range of possible $\mathrm{HC}$ environments. The ETC matrices used were generated using the following method[4]. Initially, a $\tau \times 1$ baseline column vector, $\mathrm{B}$, of floating point values is created. Let ${ }^{\omega_{b}}$ be the upper bound of the range of possible values within the baseline vector. The baseline column vector is generated by repeatedly selecting a uniform random number, $x_{b}^{i} \in\left[1, \omega_{b}\right)$, and letting $B(i)=x_{b}^{i}$ for $1 \leq i \leq \tau$. Next, the rows of the ETC matrix are constructed. Each element ETC(ti , mj) in row $i$ of the ETC matrix is created by taking the baseline value, $\mathrm{B}(\mathrm{i})$, and multiplying it by a uniform random number, $x_{r}^{i, j}$, which has an upper bound of $\omega_{r}$. This new random number, $x_{r}^{i, j} \in\left[1, \omega_{r}\right)$, is called a row multiplier. One row requires $\mu$ different row multipliers, $1 \leq j \leq \mu$. Each row $\mathrm{i}$ of the ETC matrix can then be described as $\mathrm{ETC}(\mathrm{ti}, \mathrm{mj})=$ $B(i) \times x_{r}^{i, j}$, for $1 \leq j \leq \mu$. (The baseline column itself does not appear in the final ETC matrix.) This process is repeated for each row until the $\tau \times \mu$ ETC matrix is full. Therefore, any given value in the ETC matrix is within the range $\left[1, \omega_{b} \times \omega_{r}\right)$.

To generate different mapping scenarios, the characteristics of the ETC matrix were varied based on several different methods. The amount of variance among the execution times of tasks in the metatask for a given machine is defined as task heterogeneity. Task heterogeneity is varied by changing the upper bound of the 
Learning Automata Based Algorithms for Mapping of a Class of Independent Tasks over Highly Heterogeneous Grids 9

random numbers within the baseline column vector. High task heterogeneity was represented by $\omega_{b}=3000$ and low task heterogeneity used $\omega_{b}=100$. Machine heterogeneity represents the variation that is possible among the execution times for a given task across all the machines. Machine heterogeneity was varied by changing the upper bound of the random numbers used to multiply the baseline values. High machine heterogeneity values were generated using $\omega_{r=1000 \text {, while low machine }}$ heterogeneity values used $\omega_{r=10}$. The ranges were chosen to reflect the fact that in real situations there is more variability across execution times for different tasks on a given machine than the execution time for a single task across different machines.

Different ETC matrix consistencies were used to capture more aspects of realistic mapping situations. An ETC matrix is said to be inconsistent if the ETC matrices are kept in the unordered, random state in which they were created. The ETC matrix indicates consistent characteristics if a machine $j$ executes any task $i$ faster than machine $k$, then machine $j$ executes all tasks faster than machine $k$. The consistent matrix can be obtained by sorting every row of the matrix independently. Between two special situations, a semi-consistent matrix represents a partial ordering among the machine/task execution times. For the semi-consistent matrix used here, the row elements in even columns of row $i$ are extracted, sorted and replaced in order, while the row elements in odd columns remain unordered.

Twelve combinations of ETC matrix characteristics are possible: high or low task heterogeneity, high or low machine heterogeneity, and one type of consistencies (consistent, inconsistent, or semi-consistent). Among the twelve combinations the most heterogeneous environment is modeled with inconsistent, high task and machine heterogeneous ETC, and correspondingly the least heterogeneous environment is modeled with consistent, low task and machine heterogeneous ETC. Other combinations are between these two extremes, where inconsistent ETC represents more heterogeneity than semi-consistent and consistent ETC represents less heterogeneity than semi-consistent. In charts presented in this section, Low and High task/machine heterogeneity are abbreviated to LoLo and HiHi, respectively.

The results reported here are averaged over 50 trials. All experiment results are for 200 tasks and 20 machines. The makespan for each experiment is normalized with respect to the benchmark heuristic, which is RC. Unless stated, the learning automata model used in the experiments is $\mathrm{L}_{\mathrm{RI}}$ with $a=0.01$ for algorithm A1 and $a=0.001$ for algorithms A2 and A3. For algorithms A2 and A3, the weights $\lambda_{\eta}$ and $\lambda_{\delta}$ are set to 0.4 and 0.6 , respectively, for inconsistent environment, and set to 0.05 and 0.95 for semiconsistent and consistent environments. Matching weightage is set to a very smaller value than system utilization weightage in semi-consistent and consistent environments, because in consistent environments all tasks have the same first choice for matching, the fastest machine. There is the same situation in a semi-consistent environment because of its consistent sub-matrix. Therefore, the decisive factor in gaining a better makespan is to maximize system utilization rather than matching proximity. Termination condition is met when, no change in makespan is made for 1500 consecutive iterations, or number of iterations exceeds 500000 . 


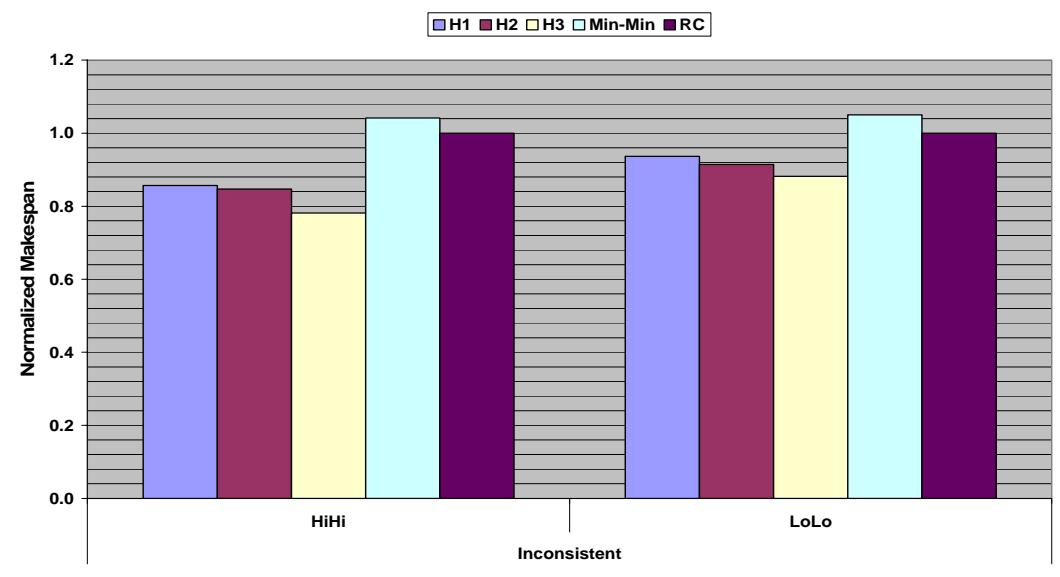

Fig. 3. Makespan for inconsistent environment

In Figure 3, three proposed algorithms are compared with Min-Min and RC in term of normalized makespan for inconsistent heterogeneity. It can be noted that all three proposed algorithms outperform both RC and Min-Min. For high machine and task heterogeneity, makespan resulted by algorithm A3 is 21 percent less than the makespans resulted from RC. Algorithm A2 performs slightly better than algorithm A1, and algorithm A3 performs better than algorithms A1 and A2. Figure 4 compares the normalized makespans of the proposed algorithms with the Min-Min and RC. All three proposed algorithms outperform Min-Min. Algorithms A1 and A3 perform better than RC for high task and machine heterogeneity; however, algorithm A2 fails to outperform RC. Except algorithm A3, the other two algorithms perform worse than $\mathrm{RC}$ for low task and machine heterogeneity.

From Figure 5, it can be stated for consistent environments, RC and Min-Min performs better than the algorithms proposed in this paper. Results shown in Figure 6 indicate the fact that the proposed algorithms perform significantly better than both $\mathrm{RC}$ and Min-Min for inconsistent environments, while they fails to perform better than RC and Min-Min for consistent environment. For semi-consistent environment whose heterogeneity is between consistent and inconsistent, learning automata outperforms Min-Min, but performs very closely to RC. Therefore, proposed algorithms perform better in environments with higher level of heterogeneity.

As expected, algorithm A3 performs better than algorithm A2 because it can avoid trapping in local minimums. Observing the results of the experiments, it is evident that algorithm A1 performs very close to and even better than algorithm A2 although it has a completely different reward criterion. It is worth mentioning that in contrast to algorithms A2 and A3 which use detailed information of expected run time of each task on each machine to guide learning automata, algorithm A1 ignores such information and guide learning automata blindly. 
Learning Automata Based Algorithms for Mapping of a Class of Independent Tasks over Highly Heterogeneous Grids 11

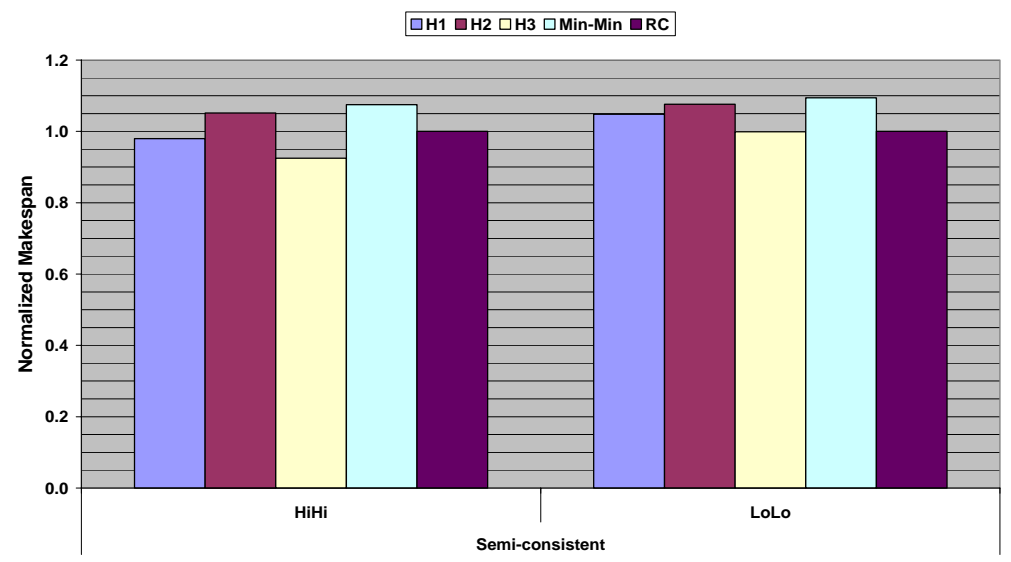

Fig. 4. Makespan for semi-consistent environment

The other important issue to consider is the computational cost of finding a mapping using each algorithm. Figure 7 shows a comparison in terms number of iterations. On average, algorithm A2 finds a mapping in about 39000 iterations, while algorithm A3 needs 12 times more iterations. It should be noted that the reward parameter $(a)$ which is set to 0.01 for algorithm A2, but 0.001 for algorithms A1 and A3 may account for faster convergence of algorithm A2 in contrast to algorithms A1 and A3. However, each algorithm is compared with others by setting learning parameter to a value that yields best result. For further experiments and analysis, interested reader may refer to [12].

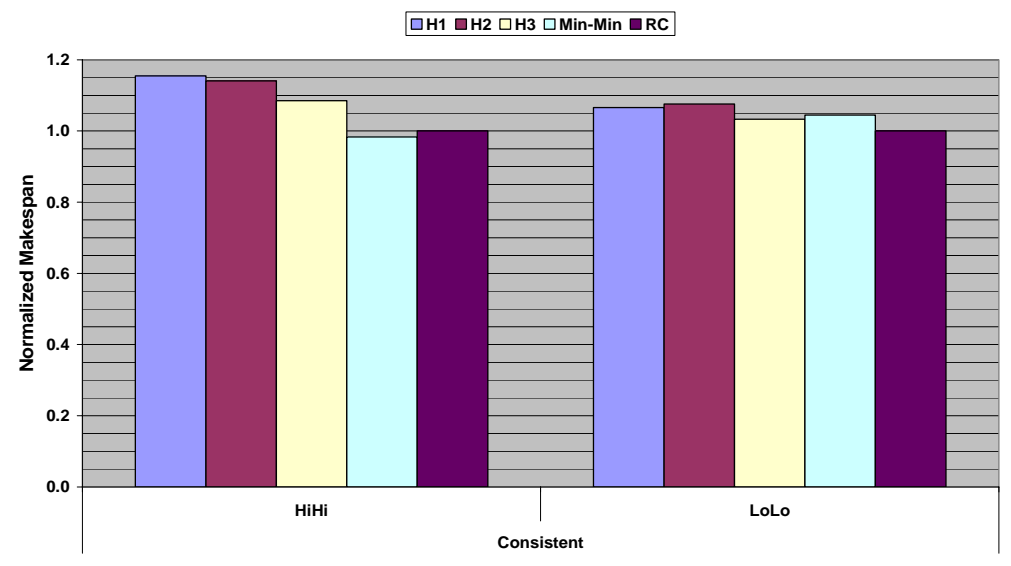

Fig. 5. Makespan for consistent environment 


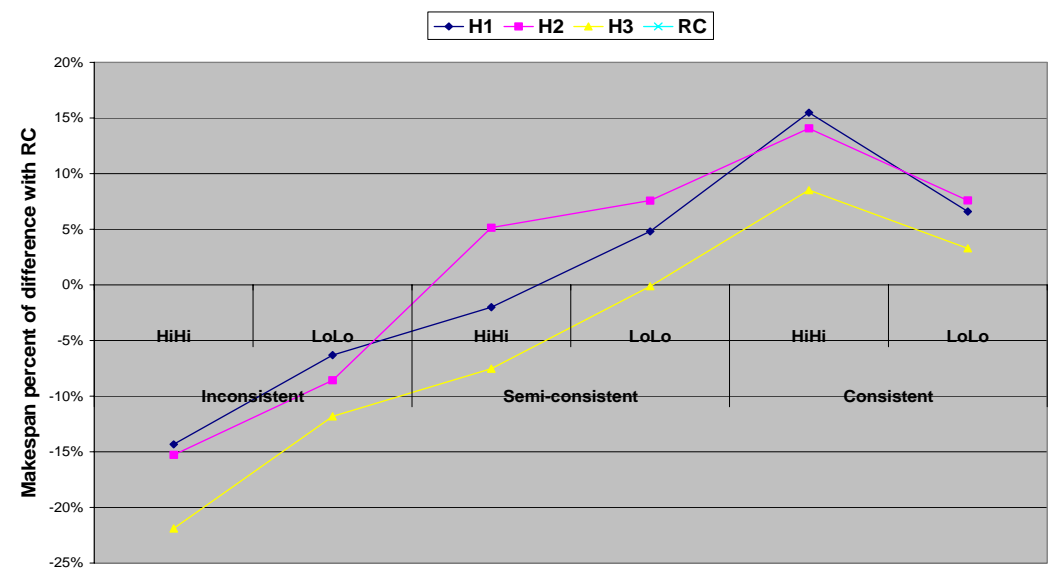

Fig. 6. Difference of makespan with RC for different consistency and heterogeneity

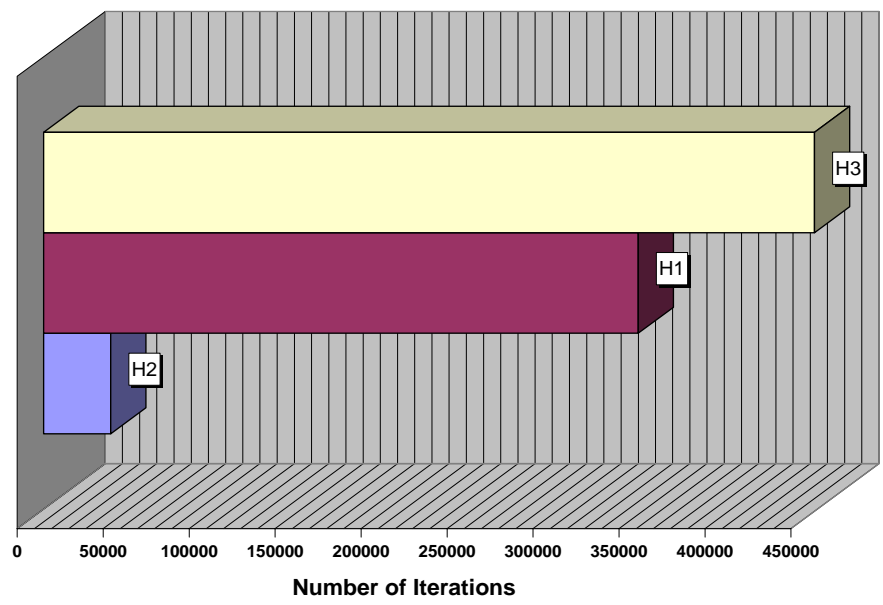

Fig. 7. Comparison of proposed algorithms with respect to number of iterations

\section{References}

[1].A. L. Rosenberg, Optimal scheduling for cycle-stealing in a network of workstations with a bag-of-tasks workload, IEEE Trans. Parallel Distributed Systems, 13(2), 2002, 179-191.

[2].H. Casanova, T.M. Bartol, J. Stiles, F. Berman, Distributing MCell simulations on the grid, Int. J. High Perform. Comput. Appl.,15 (3), 2001, 243-257.

[3].S. Smallen, W. Cirne, J. Frey, F. Berman, R. Wolski, M. Su, C. Kesselman, S. Young, M. Ellisman, Combining workstations and supercomputers to support grid applications: the 
Learning Automata Based Algorithms for Mapping of a Class of Independent Tasks over Highly Heterogeneous Grids 13

parallel tomography experience, IEEE Proceedings of the Ninth Heterogeneous Computing Workshop, May 2000, 241-252.

[4].M. Macheswaran, S. Ali, H.J. Siegel, D. Hensgen, R.F. Freund, Dynamic mapping of a class of independent tasks onto heterogeneous computing systems, J. Parallel Distribut. Comput., 59 (2), 1999, 107-131.

[5].T. D. Braun, H. J. Siegel , and N. Beck, A comparison of eleven static heuristics for mapping a class of independent tasks onto heterogeneous distributed computing systems, $J$. Parallel and Distributed Computing, 61, 2001, 810-837.

[6].T. D. Braun, H. J. Siegel, et al., Taxonomy for describing matching and scheduling heuristics for mixed-machine heterogeneous computing systems, Proceedings of the $17^{\text {th }}$ IEEE Symposium on Reliable Distributed Systems, October 1998, 330-335.

[7].Min-You $\mathrm{Wu}$ and Wei Shu, A high-performance mapping algorithm for heterogeneous computing systems, Proceedings of 15th International Parallel and Distributed Processing Symposium (IPDPS'01), April 2001.

[8].K. Narendra and M. A. L. Thathachar. "Learning Automata: An Introduction," Prentice Hall, Englewood Cliffs, New Jersey, 1989.

[9].F. Berman, "High-performance schedulers," in The Grid: Blueprint for a New Computing Infrastructure, I. Foster and C Kesselman, eds., Morgan Kaufmann, San Francisco, CA, 1999, 279-310.

[10].H. Chen and M. Maheswaran, Distributed dynamic scheduling of composite tasks on grid computing systems, Proceedings of Int'l parallel and distributed Processing Symposium (IPDPS'02), 2002.

[11].O. H. Ibarra and C. E. Kim, Heuristic Algorithms for scheduling independent tasks on non-identical processors, J. ACM, 24(2), April 1977, 280-289.

[12].S. Ghanbari and M. R. Meybodi, Various mapping algorithms for heterogeneous computational grid: learning automata approach, Technical Report, Computer Engineering Department and Information Technology, Tehran, Iran, 2004.

[13]. C. Weng and X. Lu, Heuristic scheduling for bag-of-tasks applications in combination with QoS in the computational grid, J. Future Generation Computer Systems, 2003 